\title{
Straight cracks in circular positions in plane elasticity
}

\begin{abstract}
A system of hypersingular integral equation for the multiple straight cracks in a circular position in plane elasticity is formulated and presented. The center of the cracks are placed at the edge of a circle with radius $\mathrm{R}$. The second crack in this problem is located in a different position based on the varying angle, $\mathrm{d}$. The sraight cracks problem is reduced to a system of hypersingular integral equations by using the method of complex potential. With the help of particular quadrature rules, the unknown coefficients are solved numerically from the resulting system of hypersingular integral equations. The obtained unknown coefficients are then can be used for determining the stress intensity factor (SIF). To prove that the suggested method can be used to solve more complicated model cases of the cracks in circular positions, examples are given to demonstrate the behaviour of SIF for different crack positions.
\end{abstract}

Keyword: Straight cracks; Circular positions; Plane elasticity 